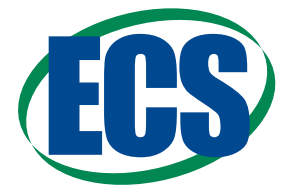

\title{
Discharge, Relaxation, and Charge Model for the Lithium Trivanadate Electrode: Reactions, Phase Change, and Transport
}

\author{
Nicholas W. Brady, ${ }^{a}$ Qing Zhang, ${ }^{\text {b }}$ K. W. Knehr, ${ }^{\text {a,* }}$ Ping Liu, ${ }^{c}$ Amy C. Marschilok,, ,d,** \\ Kenneth J. Takeuchi, ${ }^{\mathrm{b}, \mathrm{d}, * *}$ Esther S. Takeuchi, ${ }^{\mathrm{b}, \mathrm{c}, \mathrm{d}, * * *}$ and Alan C. West ${ }^{\mathrm{a}, \mathrm{e}, * * *, \mathrm{z}}$ \\ ${ }^{a}$ Department of Chemical Engineering, Columbia University, New York, New York 10027, USA \\ ${ }^{b}$ Department of Chemistry, Stony Brook University, Stony Brook, New York 11794, USA \\ ${ }^{c}$ Energy Sciences Directorate, Brookhaven National Laboratory, Upton, New York 11973, USA \\ ${ }^{d}$ Department of Materials Science and Engineering, Stony Brook University, Stony Brook, New York 11794, USA \\ ${ }^{e}$ Department of Earth and Environmental Engineering, Columbia University, New York, New York 10027, USA
}

\begin{abstract}
The electrochemical behavior of lithium trivanadate $\left(\mathrm{LiV}_{3} \mathrm{O}_{8}\right)$ during lithiation, delithiation, and voltage recovery experiments is simulated using a crystal-scale model that accounts for solid-state diffusion, charge-transfer kinetics, and phase transformations. The kinetic expression for phase change was modeled using an approach inspired by the Avrami formulation for nucleation and growth. Numerical results indicate that the solid-state diffusion coefficient of lithium in $\mathrm{LiV}_{3} \mathrm{O}_{8}$ is $\sim 10^{-13} \mathrm{~cm}^{2} \mathrm{~s}^{-1}$ and the equilibrium compositions in the two phase region $(\sim 2.5 \mathrm{~V})$ are $\mathrm{Li}_{2.5} \mathrm{~V}_{3} \mathrm{O}_{8}: \mathrm{Li}_{4} \mathrm{~V}_{3} \mathrm{O}_{8}$. Agreement between the simulated and experimental results is excellent. Relative to the lithiation curves, the experimental delithiation curves show significantly less overpotential at low levels of lithiation (end of charge). Simulations are only able to capture this result by assuming that the solid-state mass-transfer resistance is less during delithiation. The proposed rationale for this difference is that the (100) face is inactive during lithiation, but active during delithiation. Finally, by assuming non-instantaneous phase-change kinetics, estimates are made for the overpotential due to imperfect phase change (supersaturation).

(C) The Author(s) 2016. Published by ECS. This is an open access article distributed under the terms of the Creative Commons Attribution Non-Commercial No Derivatives 4.0 License (CC BY-NC-ND, http://creativecommons.org/licenses/by-nc-nd/4.0/), which permits non-commercial reuse, distribution, and reproduction in any medium, provided the original work is not changed in any way and is properly cited. For permission for commercial reuse, please email: oa @electrochem.org. [DOI: 10.1149/2.0341614jes] All rights reserved.

(cc) BY-NC-ND
\end{abstract}

Manuscript submitted August 31, 2016; revised manuscript received October 3, 2016. Published October 26, 2016.

Large scale transportation and stationary applications of lithium ion batteries require inexpensive, reliable, and safe systems. ${ }^{1}$ Transition metal (cobalt, iron, nickel, manganese, vanadium, titanium, tungsten, and molybdenum) oxides are attractive lithium intercalation cathode materials for these applications because of their natural abundance and high redox potentials. ${ }^{2}$ Conventional anode materials, such as graphite, typically have higher specific capacities than cathode materials, such as $\mathrm{LiCoO}_{2}$ and $\mathrm{LiFePO}_{4}$. This difference in capacity is because typical cathode materials can only accept one lithium per formula unit; therefore, there is a potential breakthrough in developing cathode materials that are able to host lithium-ions in excess of one per formula unit. For example, $\mathrm{LiV}_{3} \mathrm{O}_{8}$ is an attractive material because of its high potential suitable for battery applications $(\sim 3 \mathrm{~V})$ and high theoretical specific energy $\left(\sim 374 \mathrm{mAh} \mathrm{g}^{-1}\right) .^{3-5}$ The high capacity comes from the ability of the matrix to host three additional (excess) lithium ions $\left(\mathrm{Li}_{4} \mathrm{~V}_{3} \mathrm{O}_{8}\right){ }^{6}$

Because $\mathrm{LiV}_{3} \mathrm{O}_{8}$ is a promising mid-voltage material with high capacity and good cycling ability, it has received research attention. However, despite this attention, important physical parameters such as the diffusion coefficient of lithium in the material are not known with precision, varying by at least two orders of magnitude. ${ }^{7,8}$ In addition, the material undergoes a phase change at $\sim 2.5 \mathrm{~V}$ from $\mathrm{Li}_{1+\mathrm{x}} \mathrm{V}_{3} \mathrm{O}_{8}$ to $\mathrm{Li}_{4} \mathrm{~V}_{3} \mathrm{O}_{8},{ }^{6,9,10}$ but the equilibrium composition, specifically in the lithium-deficient phase is not known with precision ${ }^{11}$ and, to the authors' knowledge, there are no studies on the kinetics of phase change in this material.

Through the development of a continuum model, this paper seeks to quantify the diffusion coefficient as well as the parameters governing phase change in lithium trivanadate. In other materials, several models have been proposed to account for phase change, including shrinking-core, mosaic, domino-cascade, and core-shell models..$^{12-15}$ The shrinking-core model is the most commonly used model because it gives good agreement with electrochemical measurements, it is intuitive, and numerically robust. Conceptually, this method is limited

\footnotetext{
*Electrochemical Society Student Member.

**Electrochemical Society Member.

$* * *$ Electrochemical Society Fellow.

zE-mail: acw7@columbia.ed
}

because it generally assumes instantaneous phase transformation kinetics. Because we seek to quantify the phase change kinetics, the shrinking-core method is of little use.

In order to quantify the kinetics of the phase transition, this paper develops a model which accounts for lithium diffusion and phase change reaction as parallel processes. Depending on parameter values, this model can replicate a shrinking-core model, yet it is easier to implement. The model retains the main advantages of the shrinkingcore model: agreement with electrochemical measurements, intuitive, numerically robust, and has the added advantage that it does not require the tracking of moving interior boundaries. This model utilizes the Avrami treatment of nucleation and growth to describe phase change. ${ }^{16-18}$ While a description of nucleation and growth has been used previously to describe phase change in battery materials, ${ }^{19,20}$ this work is the first to validate a continuum model using Avrami kinetics against electrochemical measurements taken during discharge, charge, and relaxation.

\section{Experimental}

Materials synthesis and characterization.- $-\mathrm{Li}_{1.1} \mathrm{~V}_{3} \mathrm{O}_{8}$ materials were prepared via a sol-gel approach. ${ }^{21}$ Briefly, $\mathrm{LiOH} \cdot \mathrm{H}_{2} \mathrm{O}$ and $\mathrm{V}_{2} \mathrm{O}_{5}$ in a stoichiometric ratio of $\mathrm{Li}: \mathrm{V}=1.1: 3$ were used as starting materials and stirred in $50^{\circ} \mathrm{C}$ aqueous solution under $\mathrm{N}_{2}$ atmosphere for $24 \mathrm{~h}$ followed by freeze-drying. The precursor materials were heat-treated at $550^{\circ} \mathrm{C}$ to get the final product. SEM images were taken with an accelerating voltage of $10 \mathrm{kV}$ on a JEOL 7600F Field Emission Scanning Electron Microscopy at the Center of Functional Nanomaterials at Brookhaven National Laboratory.

Electrochemical measurements. $-\mathrm{Li}_{1.1} \mathrm{~V}_{3} \mathrm{O}_{8}$ cathodes were prepared by mixing $\mathrm{Li}_{1.1} \mathrm{~V}_{3} \mathrm{O}_{8}$ powders, carbon, graphite, and polyvinylidene fluoride (85:5:5:5 weight percent, respectively) in N-Methyl-2pyrrolidone solution and the slurry was cast onto Al foil, with an active material loading of $3.4 \pm 0.1 \mathrm{mg} \mathrm{cm}^{-2}$. Coin cells were assembled in an Argon-filled glove box with lithium-metal as the anode and $1 \mathrm{M} \mathrm{LiPF}_{6} \mathrm{EC}$ (ethylene carbonate)/DMC (dimethyl carbonate) (volume ratio 3:7) as electrolyte. Galvanostatic cycling tests were carried out on Maccor Battery Test Equipment at $\mathrm{C} / 10$ to 1.9 electron equivalents or at $\mathrm{C} / 5, \mathrm{C} / 2$ and $1 \mathrm{C}$ rate to $2.4 \mathrm{~V}$ (specific current of $37.49,74.98,182.8$, and $360.6 \mathrm{~mA} \mathrm{~g}^{-1}$ respectively). The current 


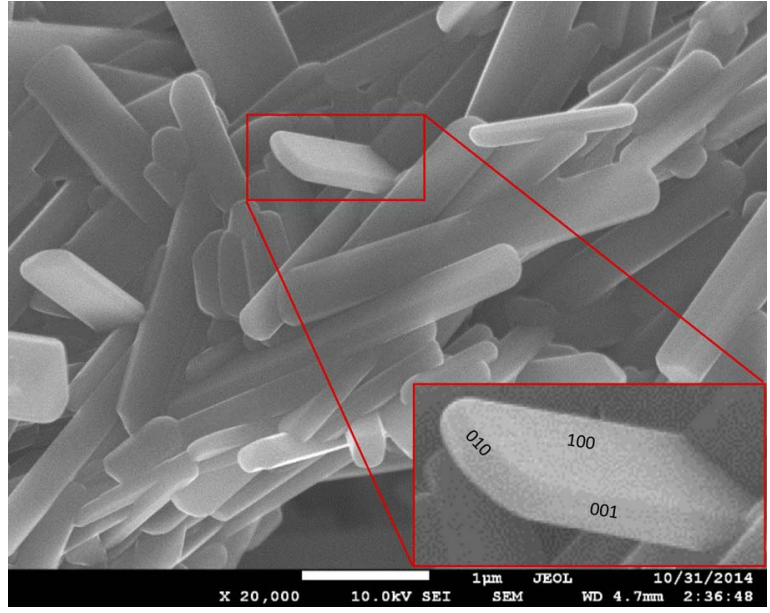

Figure 1. SEM images of $\mathrm{LiV}_{3} \mathrm{O}_{8}$ crystals sintered at $550^{\circ} \mathrm{C}$ for 2 hours. The inset highlights a representative crystal with the three faces labeled with their respective planes.

density for the coin cells was precisely controlled to be proportional to $\mathrm{C}$ rate $\left(0.126,0.253,0.632,1.26 \mathrm{~mA} \mathrm{~cm}^{-2}\right.$ respectively). While efforts were made to produce cells with similar active material loadings, the loadings between cells were not identical, therefore the $\mathrm{C}$ rates are approximate.

Ab-Initio theory calculations.-DFT calculations were performed within the Generalized Gradient Approximation (GGA) using the PW91 ${ }^{22}$ pseudo-potential as implemented in VASP. ${ }^{23} \mathrm{~A}+U$ correction term was used for $\mathrm{V}$. The exact $U$ values we used in this work were cited from fitting enthalpy of formation for binary oxides. ${ }^{24} \mathrm{LiV}_{3} \mathrm{O}_{8}$ surfaces were modeled by two $\mathrm{Li}_{4} \mathrm{~V}_{12} \mathrm{O}_{32}$ layers, where the bottom $\mathrm{Li}_{4} \mathrm{~V}_{12} \mathrm{O}_{32}$ layers in the unit cell was fixed in their optimized bulk positions while the top layer was allowed to relax. The Brillouinzone integration was performed on a grid of $2 \times 3 \times 1$ MonkhorstPack $^{25}$ special k-points. A vacuum layer of $20 \AA$ thick was applied perpendicular to the slab to avoid artificial interactions between the slab and its periodic images.

Experimental Results.-From the SEM images of the synthesized $\mathrm{LiV}_{3} \mathrm{O}_{8}$ crystals, the particle dimensions were measured using ImageJ software. Measuring many of these crystals gives order of magnitude estimations for the dimensions: $10-100 \mathrm{~nm}, 1000 \mathrm{~nm}, 100 \mathrm{~nm}$ for the [100], [010], and [001] directions respectively.

Figure 2 is a discharge curve conducted at a $\mathrm{C} / 10$ current rate $\left(37.49 \mathrm{~mA} \mathrm{~g}^{-1}\right)$ to a depth of $x=1.9$ in $\mathrm{Li}_{1+\mathrm{x}} \mathrm{V}_{3} \mathrm{O}_{8}(176.82 \mathrm{mAh}$ $\left.\mathrm{g}^{-1}\right)$. The voltage plateau at about $2.5 \mathrm{~V}$ suggests a two-phase region, as has been documented previously. ${ }^{6,9,10}$ At the end of discharge, the current is interrupted and voltage recovery is measured. The recovered voltage is composed of a charge-transfer overpotential, $\eta_{C T}$, and a mass-transfer overpotential, $\eta_{M T}$, associated with non-uniformities of the solid-state lithium concentration within the crystal. It is seen that the charge-transfer losses recover nearly instantaneously, while the mass-transfer overpotential relaxes over longer time periods (on the order of an hour). It is important to understand on which length scales these mass-transfer losses are occurring because it informs us which processes are performance limiting, from which we can improve electrode design. Using the bulk electrode thickness and bulk diffusion coefficient, relaxation is expected to occur on the order of a minute, $\tau=\frac{L^{2}}{D}=\frac{(0.005 \mathrm{~cm})^{2}}{\left(10^{-6} \mathrm{~cm}^{2} \mathrm{~s}^{-1}\right)}=25 \mathrm{~s}$. This suggests that mass-transport within the electrolyte is not performance limiting.

The $\mathrm{LiV}_{3} \mathrm{O}_{8}$ crystal has a layered structure, ${ }^{8,11}$ where intralayer transport of lithium, along the [010] and [001] directions, is preferred over interlayer transport, the [100] direction. ${ }^{26,27}$ Assuming that transport in the [100] direction is negligible compared to transport in the other directions, and assuming that rates of transport in the [010] and

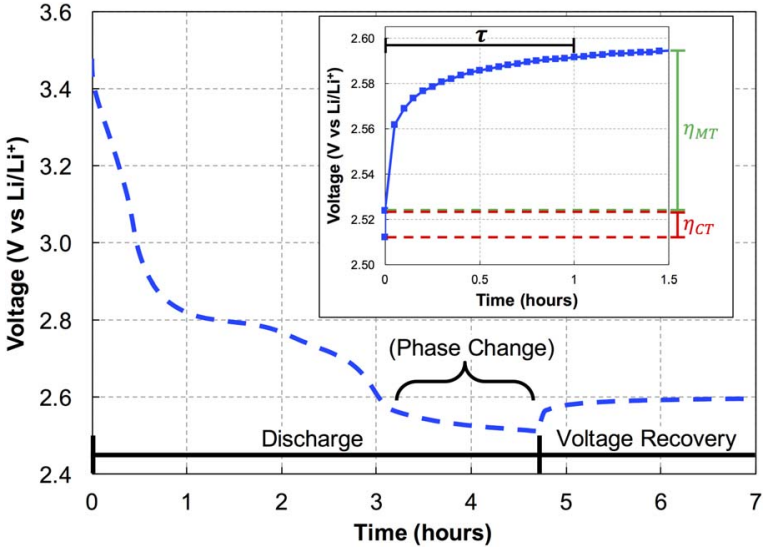

Figure 2. Representative potential curve during discharge and recovery. The constant voltage plateau seen during the discharge indicates a two-phase region. The voltage recovery (inset) is divided into charge-transfer losses $\left(\eta_{C T}\right)$ and mass-transfer losses $\left(\eta_{M T}\right)$. The characteristic relaxation time, $\tau$, is also determined from the voltage recovery data.

[001] directions are approximately equal, it is hypothesized that diffusion in the [001] direction is performance limiting. Using the crystal thickness in the [001] direction and the solid-state diffusion coefficient, we expect the characteristic diffusion time (relaxation time) to be on the order of an hour, $=\frac{L^{2}}{D}=\frac{(100 \mathrm{~nm})^{2}}{\left(10^{-13} \mathrm{~cm}^{2} \mathrm{~s}^{-1}\right)}=1000 \mathrm{~s}$. Although Figure 1 shows that the crystals agglomerate, the dimensional analysis suggests that mass transfer losses are dominated by diffusion resistances on the crystal scale. To further test this hypothesis a mathematical model was developed.

\section{Theory}

A qualitative comparison of the shrinking-core and nucleation and growth models is given in Figure 3. For both formulations, lithium
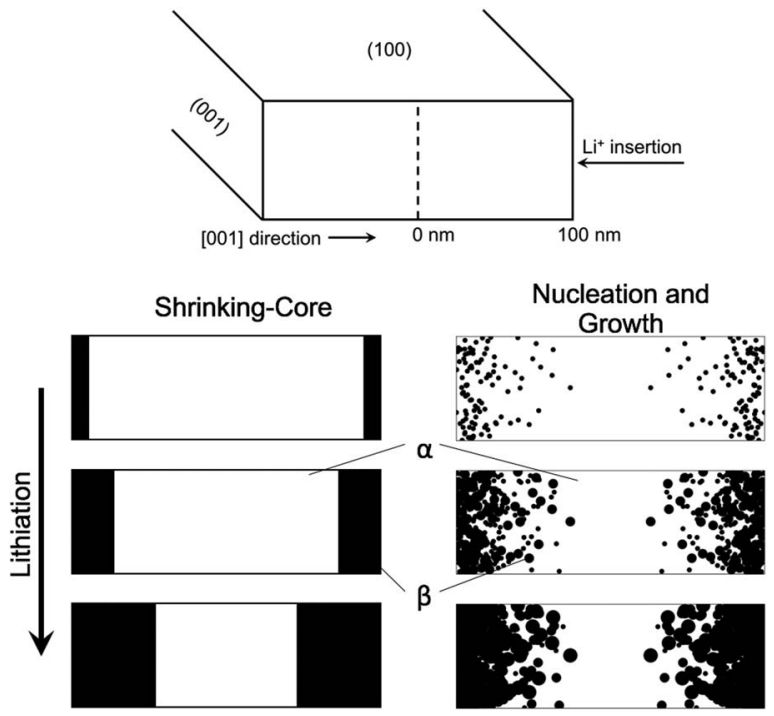

Figure 3. (Top) Schematic of the modeling domain. It is assumed that $\mathrm{Li}^{+}$predominantly inserts through the (001) crystal face and therefore concentration variations only occur along the [001] direction. (Bottom) Qualitative representations of two models for phase-change are compared: the commonly utilized shrinking-core method and the nucleation and growth model. As lithium is inserted into the material, the concentration of lithium increases. Once the concentration of lithium exceeds the saturation value, phase change commences, and a new lithium rich $\beta$-phase is created. As more lithium is inserted, the amount of $\beta$-phase increases. The shrinking-core method assumes that the new phase forms in a layer by layer process, while the nucleation and growth model produces a profile that is similar, but with a more dispersed $\beta$-phase. 
inserts into the active material, and below a threshold concentration, $c_{\alpha, s a t}$, there is no phase change. However once the local concentration of lithium in the $\alpha$-phase exceeds $c_{\alpha, s a t}$, the material phase separates into a lithium-deficient $\alpha$-phase and a lithium-rich $\beta$-phase as follows:

$$
L i_{1+x} V_{3} O_{8} \rightarrow y L i_{1+x_{\alpha, s a t}} V_{3} O_{8}+(1-y) L i_{1+x_{\beta}, s a t} V_{3} O_{8}
$$

At equilibrium the molar ratio of $\alpha$-phase to $\beta$-phase can be calculated:

$$
y=\frac{x_{\beta, s a t}-x}{x_{\beta, s a t}-x_{\alpha, s a t}} ; \quad x_{\alpha, s a t}<x<x_{\beta, s a t}
$$

Following the development outlined by Knehr et al. ${ }^{28,29}$ the hypothesis of phase formation was further explored by developing a nucleation and growth model on the crystal scale with the following assumptions:

1. The system is isothermal.

2. The impact of intermolecular interactions on solute species transport is ignored (dilute solution theory).

3. Variations in concentration and potential on the bulk electrode scale and agglomerate scale are negligible.

4. The $\mathrm{LiV}_{3} \mathrm{O}_{8}$ crystals are considered to be rectangular prisms and have a constant volume.

5. The polarization associated with the lithium-metal negative electrode is negligible.

6. Only the $\alpha$-phase is electrochemically active. Lithium can only enter the $\beta$-phase by first inserting into the $\alpha$-phase, and then through a chemical reaction, enter into the $\beta$-phase.

7. Mass-transfer along the [001] direction is assumed to be rate limiting and mass-transport along the [100] and [010] directions are considered negligible.

Assumption 7 is informed from an analysis of the material's crystal structure and DFT calculations. The spacing between vanadate layers is about $6.36 \AA$, while the atoms within a vanadate layer are more close-packed. ${ }^{30,31}$ This crystal structure indicates lithium transport is anisotropic: diffusion is favored in directions parallel to the vanadate plane ([010] and [001]) and hindered in the direction normal to the plane, [100]. Using the diffusive activation energies calculated in ${ }^{27}$ (0.61 eV: $0.36 \mathrm{eV}: 0.36 \mathrm{eV}$ for the directions [100]:[010]:[001]), and the crystal aspect ratios obtained from the SEM image (Figure 1), it would be concluded that the mass flow rates in the [100] and [001] directions would be approximately equal.

However, DFT calculations suggest that the adsorption and diffusion of $\mathrm{Li}^{+}$are preferential on the (001) surface via the unique tunnel along the [001] direction. The tunnel is constructed along the zig-zag $\mathrm{VO}_{\mathrm{x}}$ plane, which provides highly symmetric oxygen sites for $\mathrm{Li}^{+}$adsorption and diffusion. The $(001)$ face provides $\mathrm{a} \mathrm{Li}^{+}$binding energy of $-0.56 \mathrm{eV}$; accordingly, the (001) face can be anticipated to be active during lithiation. In contrast, on the (010) face, lithiation is hindered by a weakened $\mathrm{Li}^{+}$adsorption with a binding energy of $-0.08 \mathrm{eV}$, which likely results in a lower coverage. The (100) face is the most inert face during lithiation, providing an adsorptive binding energy of $0.40 \mathrm{eV}$. While the diffusive mass flow along the [100] and [001] directions may be similar, charge-transfer on the (100) face may be hindered by an unfavorable binding energy, essentially rendering the (100) face an insulator during lithiation. These factors indicate that the mass-transport process can be approximated as one-directional along the [001] direction.

The description of the lithium insertion is given by Equation 3 .

$$
\mathrm{Li}^{+}+\mathrm{e}^{-}+\Gamma \leftrightarrow \mathrm{Li} \Gamma
$$

where $\Gamma$ and $\mathrm{Li} \Gamma$ are unoccupied and occupied host sites in the crystal $\left(\Gamma=\alpha-\mathrm{LiV}_{3} \mathrm{O}_{8}\right)$.

The charge-transfer kinetics of this reaction are estimated using the Butler-Volmer kinetic expression, Equations 4 and 5.

$$
\begin{gathered}
i=i_{0}\left[\exp \left(\frac{\alpha_{a} F \eta}{R_{G} T}\right)-\exp \left(-\frac{\alpha_{c} F \eta}{R_{G} T}\right)\right] \\
i_{0}=F k_{r x n} c_{0}^{\alpha_{a}} c_{\alpha}^{\alpha_{c}}\left(c_{\alpha, \text { max }}-c_{\alpha}\right)^{\alpha_{a}}
\end{gathered}
$$

In the crystal, the conservation of mass for lithium in the $\alpha$-phase and $\beta$-phase is given by

$$
\begin{gathered}
\frac{\partial\left(\left(\theta_{\alpha}+\theta_{g b}\right) c_{\alpha}\right)}{\partial t}+\frac{\partial\left(\theta_{\beta} c_{\beta}\right)}{\partial t}=\nabla \cdot\left(D_{e f f} \nabla c_{\alpha}\right) \\
\frac{\partial \theta_{\beta}}{\partial t}=r_{\beta} \\
\theta_{\alpha}+\theta_{\beta}+\theta_{g b}=1
\end{gathered}
$$

where $c_{\alpha}$ and $c_{\beta}$ are the concentrations of lithium in the $\alpha$ and $\beta$-phases, $\theta_{\alpha}$ and $\theta_{\beta}$ are the volume fractions of the $\alpha$ and $\beta$-phases respectively, $r_{\beta}$ is the rate at which lithium enters the $\beta$-phase (leaves the $\alpha$-phase) and is discussed below, with further detail in Appendix A. $\theta_{g b}$ is the volume fraction of grain boundaries. It is assumed that some fraction of the particle is composed of voids or grain boundaries and that lithium can reside in these gaps. The void volume of the $\beta$-phase is some presumably very small fraction, $\zeta$, of the $\beta$-phase:

$$
\theta_{g b}=\zeta \theta_{\beta}
$$

It was initially hypothesized that the effective diffusion coefficient was given by $D_{e f f}=\theta_{\alpha} D_{\alpha}$, where mass transport is increasingly resistive as more $\beta$-phase formed. However, this formulation was inconsistent with experiment because it underpredicts the material's capacity when there are significant amounts of $\beta$-phase formation. Instead it was hypothesized that diffusion could take place not only through the $\alpha$-phase, but also along grain-boundaries and that these two processes could proceed in parallel.

$$
D_{e f f}=\theta_{\alpha} D_{\alpha}+\theta_{g b} D_{g b}
$$

This formulation is more consistent with experimental observations if $D_{g b} \sim 100 D_{\alpha}$ and $\zeta \sim 0.01$.

Boundary and initial conditions.-At the beginning of the simulation, the values of $c_{\alpha}$ and $\theta_{\beta}$ are set to initial values:

$$
\left.c_{\alpha}\right|_{t=0}=c_{\alpha, 0} ;\left.\quad \theta_{\beta}\right|_{t=0}=\theta_{\beta, 0}
$$

For each crystal, the flux at the (001) surface is defined by the specific current density and symmetry is invoked at the center of the crystal, along the [001] direction:

$$
\begin{gathered}
\left.\frac{\partial c_{\alpha}}{\partial x}\right|_{x=L}=\frac{1}{D_{e f f}} \frac{i}{F} \\
\left.\frac{\partial c_{\alpha}}{\partial x}\right|_{x=0}=0
\end{gathered}
$$

where the current density, $i$, and specific current, $i_{a p p}$, are related by Equation 14:

$$
i=i_{\text {app }} \rho L
$$

Kinetics of phase change.-The kinetics of phase change, Equation 15 are developed from Avrami's mathematical formulation of nucleation and growth, ${ }^{16-18}$ where $k_{\beta}$ is the reaction rate constant, $c_{\alpha}$ $-c_{\alpha, \text { sat }}$ is the driving force for phase change, $\theta_{\beta}$ is proportional to the interfacial area between the $\alpha$ and $\beta$-phases, and $1-\theta_{\beta}$ is the correction for impinging nuclei. A detailed derivation is given in Appendix A. The value of $m$ changes depending on the rate of nucleation relative to that of growth and the dimensionality of growth $(1,2$, or 3-dimensional).

$$
\begin{gathered}
\frac{\partial \theta_{\beta}}{\partial t}=k_{\beta}\left(c_{\alpha}-c_{\alpha, \text { sat }}\right)\left(\theta_{\beta}^{m}\right)\left[1-\theta_{\beta}\right] \\
0 \leq m \leq 1
\end{gathered}
$$

Table I gives the value of $m$ for some scenarios, but $m$ can take on any value between 0 and 1 . Combining Equations 6, 7, and 15, and assuming 1-directional diffusion in rectangular coordinates yields

$$
\frac{\partial\left(\theta_{\alpha} c_{\alpha}\right)}{\partial t}=D_{e f f} \frac{\partial^{2} c_{\alpha}}{\partial x^{2}}-k_{\beta}\left(c_{\alpha}-c_{\alpha, \text { sat }}\right)\left(\theta_{\beta}^{m}\right)\left[1-\theta_{\beta}\right]
$$


Table I. Value of $\boldsymbol{m}$ for Different Mechanisms of Phase Change.

\begin{tabular}{cccc}
$\begin{array}{c}\text { 3-D } \\
\text { Progressive }\end{array}$ & $\begin{array}{c}3-\mathrm{D} \\
\text { Instantaneous }\end{array}$ & $\begin{array}{c}\text { 2-D } \\
\text { Instantaneous }\end{array}$ & $\begin{array}{c}\text { 1-D } \\
\text { Instantaneous }\end{array}$ \\
\hline$\theta$ & $\theta^{2 / 3}$ & $\theta^{1 / 2}$ & $\theta^{0}$
\end{tabular}

By introducing dimensionless concentration, position, and time:

$$
\bar{c}=\frac{c_{\alpha}}{c_{\alpha, s a t}} ; \quad \bar{x}=\frac{x}{L} ; \quad \tau=\frac{t}{L^{2} / D_{e f f}}
$$

Equation 16 can be recast in dimensionless form:

$$
\frac{\partial\left(\theta_{\alpha} \bar{c}\right)}{\partial \tau}=\frac{\partial^{2} \bar{c}}{\partial \bar{x}^{2}}-\psi_{T h}(\bar{c}-1) \theta_{\beta}^{m}\left(1-\theta_{\beta}\right)
$$

where $\psi_{T h}$ is the ratio of phase-transformation rate to diffusion rate, akin to the Thiele modulus,

$$
\psi_{T h}=\frac{k_{\beta} L^{2}}{D_{\text {eff }}}
$$

The boundary condition given by Equation 12 is also given in dimensionless form:

$$
\left.\frac{\partial \bar{c}}{\partial \bar{x}}\right|_{\bar{x}=1}=\bar{i}
$$

where $\bar{i}$ is a dimensionless current density:

$$
\bar{i}=\frac{L^{2} \rho}{D_{\text {eff }} c_{\alpha, s a t}} \frac{i_{a p p}}{F}
$$

As $\psi_{T h}$ approaches zero, the phase fraction $\theta_{\beta}$ is uniform across the crystal, while values approaching infinity will produce profiles resembling step functions. The shrinking-core method assumes instantaneous phase change kinetics, $\psi_{T h} \rightarrow \infty$, producing the step change seen in Figure 4. At higher values of $\psi_{T h}$ the profiles resemble the shrinking-core profile. Figure 4B illustrates the effect of $\bar{i}$ on the uniformity of the $\theta_{\beta}$-profile when $\psi_{T h}=5$. It is observed that, as with $\psi_{T h}$, increasing $\bar{i}$ increases the sharpness of the profile, but clearly $\psi_{T h}$ is a stronger indicator of the $\theta_{\beta}$-profile. As $\psi_{T h}$ is increased, the relative effect of $\bar{i}$ on the $\theta_{\beta}$-profile decreases.

With the shrinking-core method, tracking a few boundaries is manageable, but not trivial. The complexity involved in tracking many boundaries whose positions vary with time, can make the shrinkingcore method cumbersome. Additional questions arise concerning the coalescence of boundaries. With the method outlined in this paper, there is no need to introduce internal boundaries and this is important

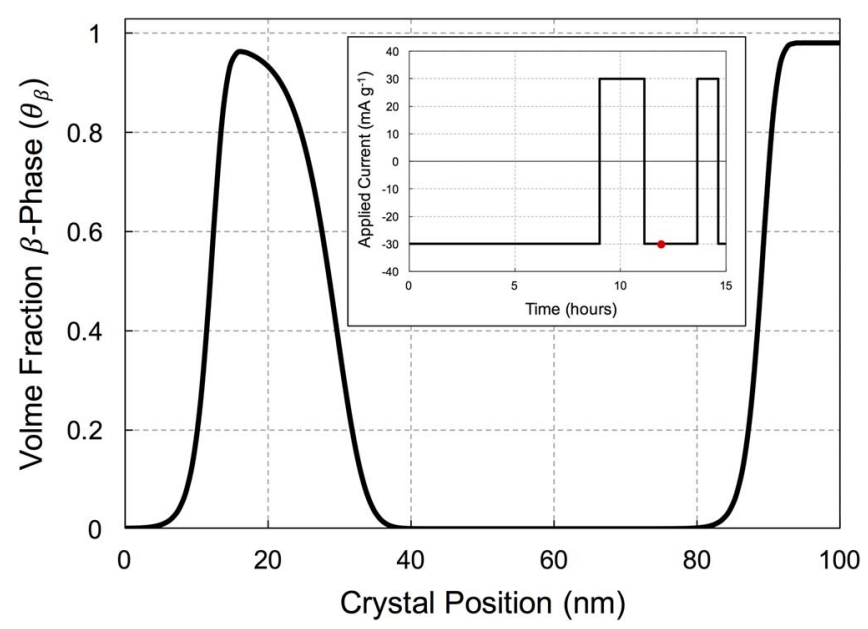

Figure 5. An illustration of the $\theta_{\beta}$ profile within a crystal $\left(\psi_{T h}=10,000, m\right.$ $=0)$ during a current cycling process $(\bar{i}=60)$. The predicted spatial variation of the regions with two phases is a strong function of the battery usage.

because simulations of battery cycling may need to account for a large number of scenarios. For example, Figure 5 shows a simulated $\theta_{\beta}$ profile, where the battery is discharged before the $\beta$-phase is completely consumed during charge. In this case, there are two distinct regions within the crystal with non-zero $\theta_{\beta}$. While qualitatively similar profiles can be simulated using the shrinking-core method, it would require separate simulations using one, two, and three internal boundaries. For ease of implementation it is desirable to find a set of governing equations that can predict the evolution of multiple boundaries without partitioning one case into multiple simulations. The authors believe the model presented here provides a practical solution to this challenge.

Numerical methods.-The governing equations were discretized using the forward-time, central-space finite volume method. The scale was discretized, and the resulting block, tri-diagonal matrix was solved in Fortran 95 using the BAND $(\mathrm{J})$ algorithm. ${ }^{32}$ The mesh size necessary to adequately resolve the profiles is dependent on the value of $\psi_{T h}$; higher values require a finer mesh. For comparison to electrochemical data, mesh sizes of 22 points were sufficient. The time step was set to 0.03 seconds. Computer experiments were conducted for the mesh sizes and time steps to ensure convergence.

The shrinking-core method commonly uses a finite volume formulation because it makes handling the internal boundary conditions
A)

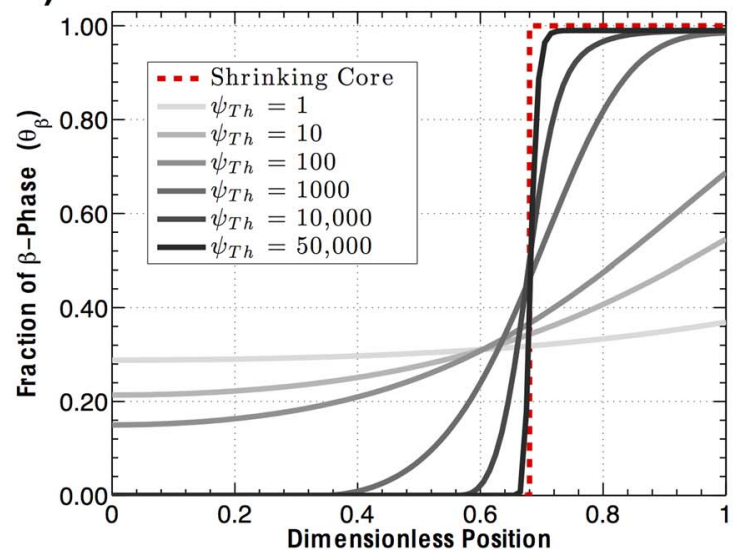

B)

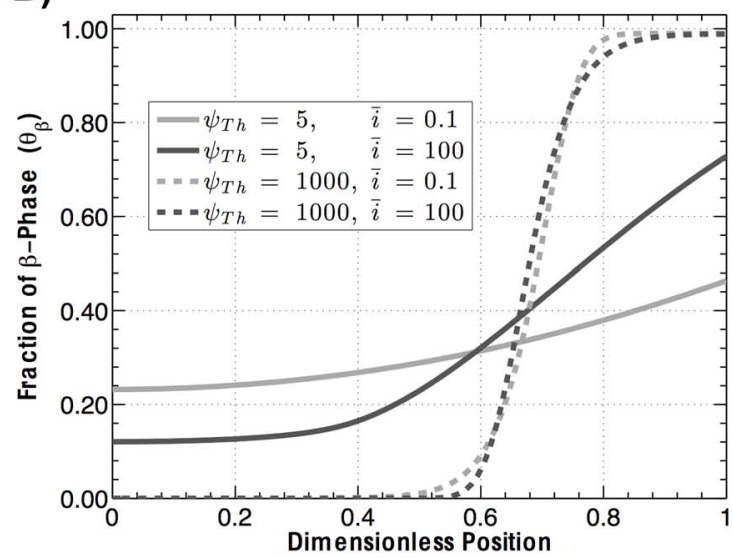

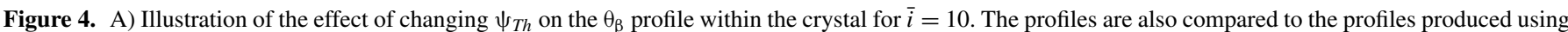

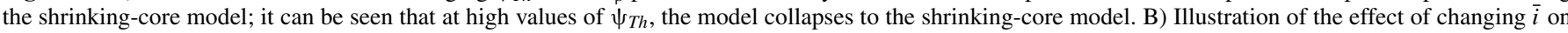
the $\theta_{\beta}$ profile within the crystal for $\psi_{T h}=5$. 
A)

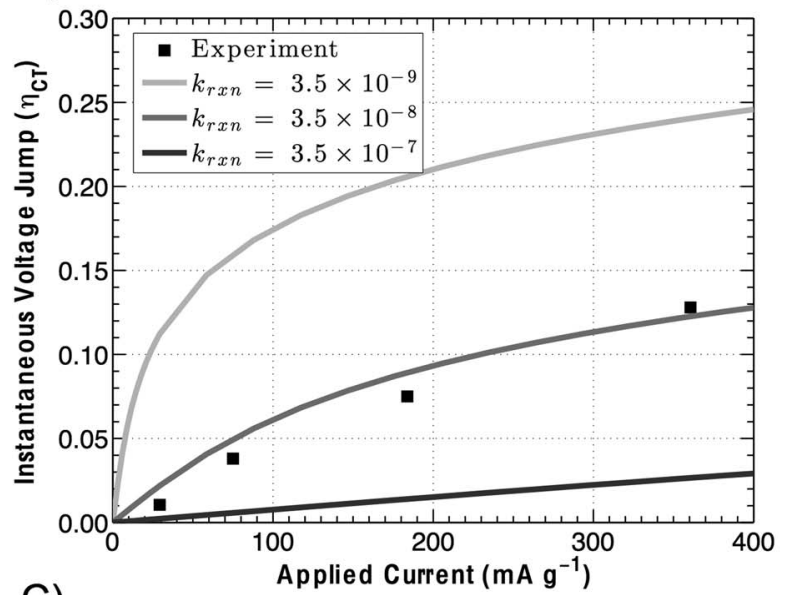

C)

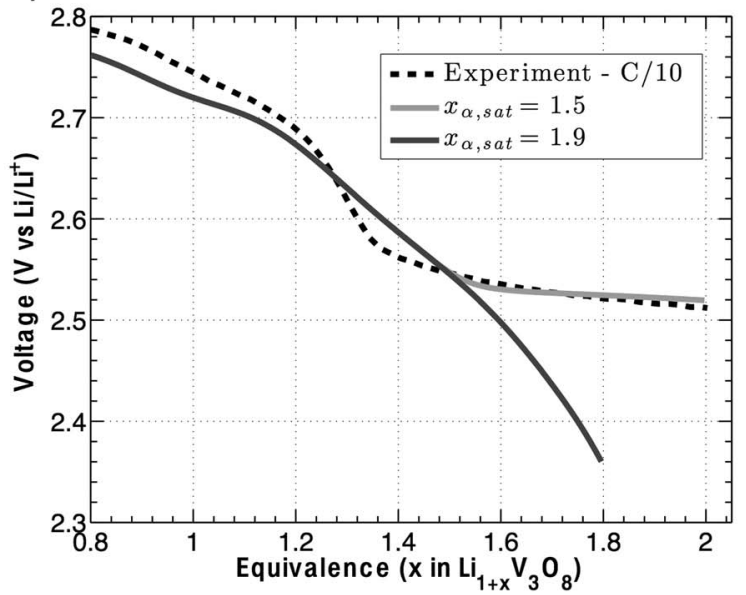

B)

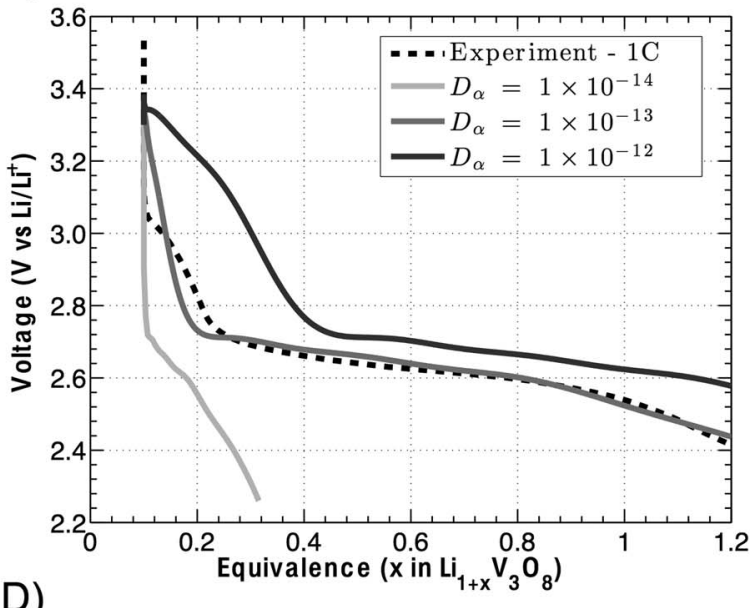

D)

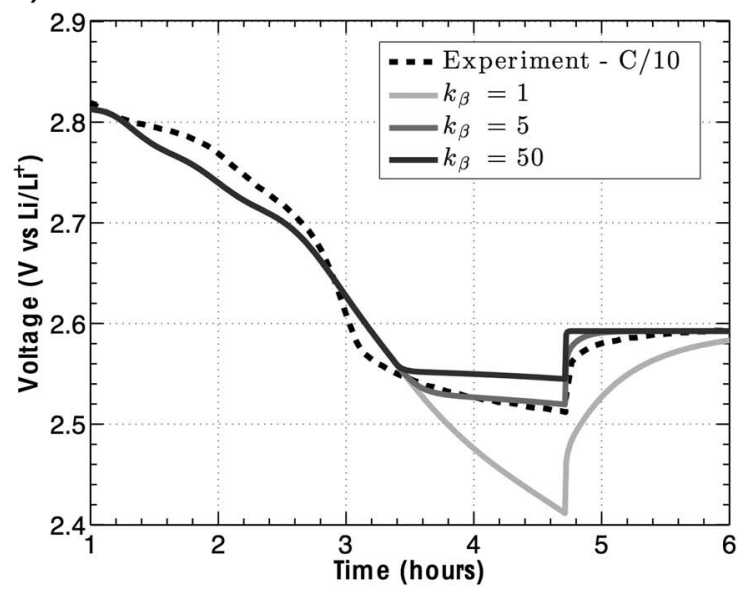

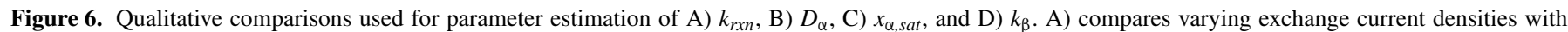

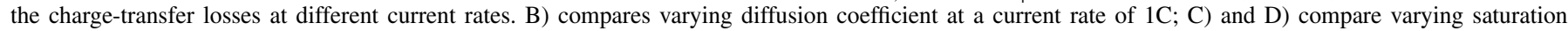
concentrations and phase change reaction coefficients, respectively, with experiments conducted at $\mathrm{C} / 10$.

more manageable. While the finite volume formulation was used to obtain the results shown in this paper, there are no significant advantages to using the finite volume method over the finite difference method; a corresponding paper treats a different electrochemical system using the finite difference method. ${ }^{33}$

\section{Parameter Estimation}

Electrodes composed of $\mathrm{LiV}_{3} \mathrm{O}_{8}$ sintered at $550^{\circ} \mathrm{C}$ were specifically chosen for study because this calcination temperature produced distinguishable crystals with clear, measurable dimensions. Other calcination temperatures, produced material which could not be readily distinguished as crystalline or amorphous and the authors could not easily determine the apparent crystallite size.

The results of the current interrupt experiments at different rates were used to get order of magnitude estimates for $k_{r x n}, D_{\alpha}$, as well as estimates for the equilibrium concentration and the rate constant for $\beta$-phase formation. From the voltage recovery data, we could elucidate information about charge-transfer and get an estimate for $k_{r x n}$ (Equation 5). The instantaneous potential jumps are the sum of the activation and ohmic overpotentials in the electrode. Assuming the ohmic losses are much smaller than the charge-transfer losses, the overpotential can be estimated using Butler-Volmer kinetics. Figure $6 \mathrm{~A}$ overlays order of magnitude estimations of $k_{r x n}$ and the experimental voltage jump observed $10 \mathrm{~ms}$ after the current is turned off at each current rate.
Using the discharge voltage versus average equivalence (6B) we are able to gain insights into the diffusive properties of lithium in $\mathrm{LiV}_{3} \mathrm{O}_{8}$. First it should be noted, that estimating the diffusion coefficient requires isolation of mass-transport effects from phase change effects, i.e. we examined the data before the onset of phase-change. In addition, at low current rates $(\mathrm{C} / 10)$ the resolution between $10^{-13}$ and $10^{-12} \mathrm{~cm}^{2} \mathrm{~s}^{-1}$ is too low to distinguish between the two conditions. However, using a higher current rate, $1 \mathrm{C}$, improves the resolution between these two cases, from which it is clearly observed that the best estimate of the diffusion coefficient is $10^{-13} \mathrm{~cm}^{2} \mathrm{~s}^{-1}$. Additionally because the value of the effective diffusion coefficient was not found to change much in the two-phase region, ${ }^{8} D_{g b}$ was assumed to be 100 times greater than $D_{\alpha}$.

Next, it was important to establish equilibrium concentrations of lithium in the $\alpha$-phase and $\beta$-phase. As previously described, the equilibrium concentration in the lithium-deficient phase was not established with certainty in the literature. Namely, it was debated whether the equilibrium concentrations were $\mathrm{Li}_{2.5} \mathrm{~V}_{3} \mathrm{O}_{8}$ : $\mathrm{Li}_{4} \mathrm{~V}_{3} \mathrm{O}_{8},{ }^{34-36}$ or as others suggest $\mathrm{Li}_{2.9-3.0} \mathrm{~V}_{3} \mathrm{O}_{8}: \mathrm{Li}_{4} \mathrm{~V}_{3} \mathrm{O}_{8} .{ }^{9,10,37}$ Because it is generally accepted that the $\beta$-phase composition is $\mathrm{Li}_{4} \mathrm{~V}_{3} \mathrm{O}_{8}, x_{\beta}$,sat was set to $3\left(c_{\beta, \text { sat }}=0.0365 \mathrm{~mol} \mathrm{~cm}^{-3}\right)$. Because the consensus in the literature is that there are only two probable possibilities for the equilibrium concentration in the $\alpha$-phase, it is relatively easy for us to test the two cases and compare the results, which are given in Figure 6C. Clearly, $x_{\alpha, \text { sat }}=1.5\left(c_{\alpha, \text { sat }}=0.0182 \mathrm{~mol} \mathrm{~cm}{ }^{-3}\right)$, or $\mathrm{Li}_{2.5} \mathrm{~V}_{3} \mathrm{O}_{8}: \mathrm{Li}_{4} \mathrm{~V}_{3} \mathrm{O}_{8}$ gives better agreement with experimental data. While some may contend that the value of $x_{\alpha, s a t}$ needs to be fitted with the value of $k_{\beta}$, this is 
simply not true for this particular case. While it is true that some nonzero value of $k_{\beta}$ needed to be selected, the value of $k_{\beta}$ only controls the slope of the voltage plateau, while $x_{\alpha, s a t}$ can be thought of as controlling the vertical position (the analog of the "y-intercept"). Using this reasoning, $x_{\alpha, \text { sat }}$ and $k_{\beta}$ can (and should) be determined independently for this case.

Figure 6D shows a comparison of experimental measurements with simulations incorporating phase change with varying rate constants, $k_{\beta}$. It can be appreciated that as $k_{\beta}$ is increased from 1 to 50 $\mathrm{cm}^{3} \mathrm{~mol}^{-1} \mathrm{~s}^{-1}$, the slope of the voltage plateau decreases, and the performance increases. The performance increase is due to decreases in the concentration overpotentials. As $k_{\beta}$ is increased, the voltage is also able to recover more quickly. The parameter $k_{\beta}$ was selected to obtain the best agreement with experimental data during the voltage plateau as well as during the voltage recovery. The reaction rate constant, $k_{\beta}$, was found to be $5.0 \times 10^{-3} \mathrm{~cm}^{3} \mathrm{~mol}^{-1} \mathrm{~s}^{-1}$, yielding $\psi_{T h}=5$. The reader should note that the parameter $m$, Equation 15, may also be fit with $k_{\beta}$.

Values of $m$ not equal to 0 , contribute to a shallow local voltage minimum at intermediate values of capacity. Because these local voltage minima were not observed experimentally and because of the fast phase change kinetics observed in the $\mathrm{LiV}_{3} \mathrm{O}_{8}$ electrode, $m=0$ was concluded to be the best estimation. The value of $m=0$ corresponds to 1-dimensional growth and instantaneous nucleation, and numerical simulations show that $m=0$ agrees well with experimental data. New phases have been observed to grow through one-dimensional growth and instantaneous nucleation in electrode materials with similar structures. ${ }^{38}$ Other values of $m$ seem to be in better agreement with other electrode materials. ${ }^{33}$

Because we have adjusted five parameters: the charge-transfer rate constant, the diffusion coefficient, the saturation concentration, the phase change rate constant, as well as the dimensionality of nucleation and growth to achieve the model-experimental fits in Figure 6, a discussion about the validity of the parameters estimated is necessary. First, the charge-transfer rate constant and diffusion coefficient can be estimated independently, therefore we believe those estimates to be reasonable. In addition, the diffusion coefficient suggested by Figure $6 \mathrm{~B}$ is within the range reported in the literature, $10^{-11}-10^{-15} \mathrm{~cm}^{2}$ $\mathrm{s}^{-1} .8$ The remaining two parameters, $x_{\alpha, \text { sat }}$ and $k_{\beta}$, could not be selected without first establishing the diffusion coefficient.

Concerning the saturation composition, the tested compositions are supported by experimental literature. Additionally, DFT calculations indicate that the saturation composition in the $\alpha$-phase is $\mathrm{Li}_{2.5} \mathrm{~V}_{3} \mathrm{O}_{8} \cdot{ }^{27}$ In summary, experiments, theoretical calculations, and this continuum model all suggest the saturation composition in the $\alpha$-phase is $\mathrm{Li}_{2.5} \mathrm{~V}_{3} \mathrm{O}_{8}$.

Finally, this brings us to the selection of the phase change kinetic parameters. First, it should be noted that changing the value of $k_{\beta}$ does not impact the experiment-model agreement before the onset of phase change, above $2.5 \mathrm{~V}$. While it cannot be said that $k_{\beta}$ is determined independently from the previously fitted parameters, the foundation of the diffusion coefficient and saturation concentration in experimental data lends credence to the validity of the selected phase change kinetic constant.

A summary of all the selected model parameters is given in Table II.

\section{Results}

Figure 7 shows the experimental and simulated voltage during discharge and recovery at current rates of $\mathrm{C} / 10, \mathrm{C} / 5, \mathrm{C} / 2$, and $1 \mathrm{C}$. Maintaining the same parameter values for each experiment, the simulations accurately predict the voltage plateau $(\sim 2.5 \mathrm{~V})$ as well as the transient and the final resting voltage during voltage recovery.

Figure 7 appears to validate the model during discharge and voltage recovery; it is also important to validate the model during charge. Figure 8A shows the experimental charge data and Figure 8B shows the simulated charge experiments. The agreement between simulation and experiment is good until the end of charge (low values of capacity), where the voltage rapidly increases with decreasing capacity.
Table II. Parameters for the $\mathrm{LiV}_{3} \mathrm{O}_{8}$ Electrode.

\begin{tabular}{lc} 
Parameter & Value \\
\hline$L(\mathrm{~nm})$ & 100 \\
$D_{\alpha}\left(\mathrm{cm}^{2} \mathrm{~s}^{-1}\right)$ & $1 \times 10^{-13}$ \\
$D_{g b}\left(\mathrm{~cm}^{2} \mathrm{~s}^{-1}\right)$ & $1 \times 10^{-11}$ \\
$\zeta$ & 0.01 \\
$\rho\left(\mathrm{g} \mathrm{cm}^{-3}\right)$ & 3.5 \\
$c_{\alpha, \text { sat }}\left(\mathrm{mol} \mathrm{cm}^{-3}\right)$ & 0.0182 \\
$c_{\beta, \text { sat }}\left(\mathrm{mol} \mathrm{cm}^{-3}\right)$ & 0.0365 \\
$k_{r x n}\left(\mathrm{~cm}^{5 / 2} \mathrm{~mol}^{-1 / 2} \mathrm{~s}^{-1}\right)$ & $3.5 \times 10^{-8}$ \\
$k_{\beta}\left(\mathrm{cm}^{3} \mathrm{~mol}^{-1} \mathrm{~s}^{-1}\right)$ & $5.0 \times 10^{-3}$ \\
$m$ & 0
\end{tabular}

In the circled region, the experimental voltage profiles collapse onto each other, suggesting smaller overpotentials during delithiation than lithiation. Simulations were conducted assuming no charge-transfer losses, but this still could not produce the observed trend. Experimental trends were captured in the simulations by increasing the diffusion coefficient during charge by a factor of 5 , Figure 9B.

The question is then, why are there differences between lithiation and delithiation? In assumption 7 it was asserted that concentration variations in the [100] direction were negligible, in part because of the lower mobility in the [100] direction and in part because chargetransfer at the (100) face was unfavorable due to a relatively low binding energy for $\mathrm{Li}^{+}$(this assumes a mechanism requiring surface adsorption prior to insertion). Possibly, the low sticking probability of $\mathrm{Li}^{+}$on the (100) face may limit the lithiation and may enhance the delithiation rate. If indeed the (100) face is active during delithiation, diffusion may proceed in all directions, albeit with a significantly lower mobility perpendicular to the (100) face. Alternatively, diffusion perpendicular to the (100) face may occur primarily through grain boundaries.

Independent of the precise mechanism, comparisons between experiments (9A) and simulations (9B) during discharge and charge are in excellent agreement by assuming a $5 \times$ increase in an effective diffusion coefficient during charge. Simulations suggest that enhanced activity of the (100) face impacts delithiation NOT by reducing the charge-transfer resistance, but instead by allowing an alternative masstransfer path for the solid-state lithium to exit the crystal. In other words, transport anisotropy is not required to justify the model during

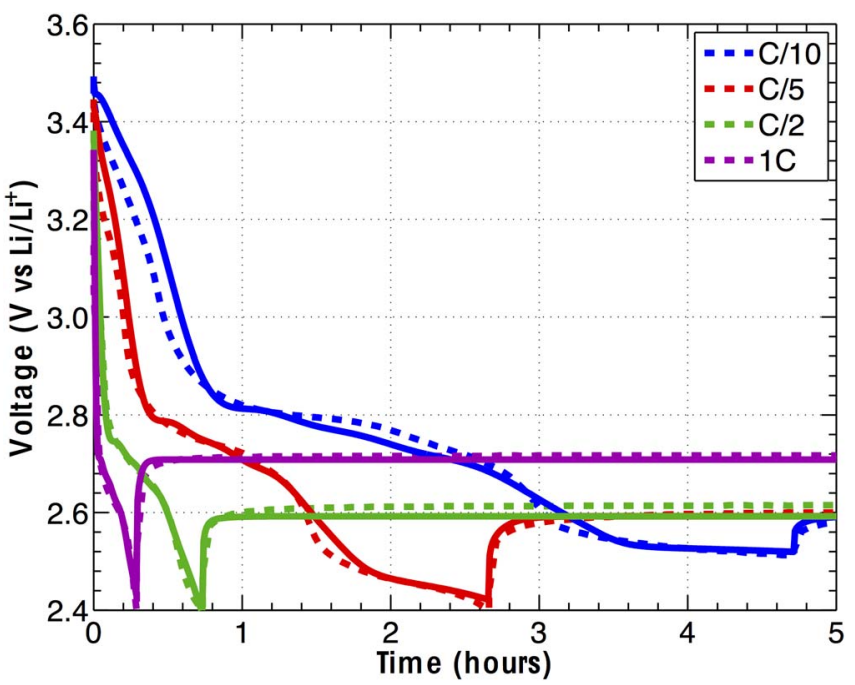

Figure 7. Experimental (dashed lines) and simulated (solid lines) potential during discharge (lithiation) and after interruption of current. Comparisons are shown for four discharge rates. The rapid change in slope of the curves is the result of current interruption. 

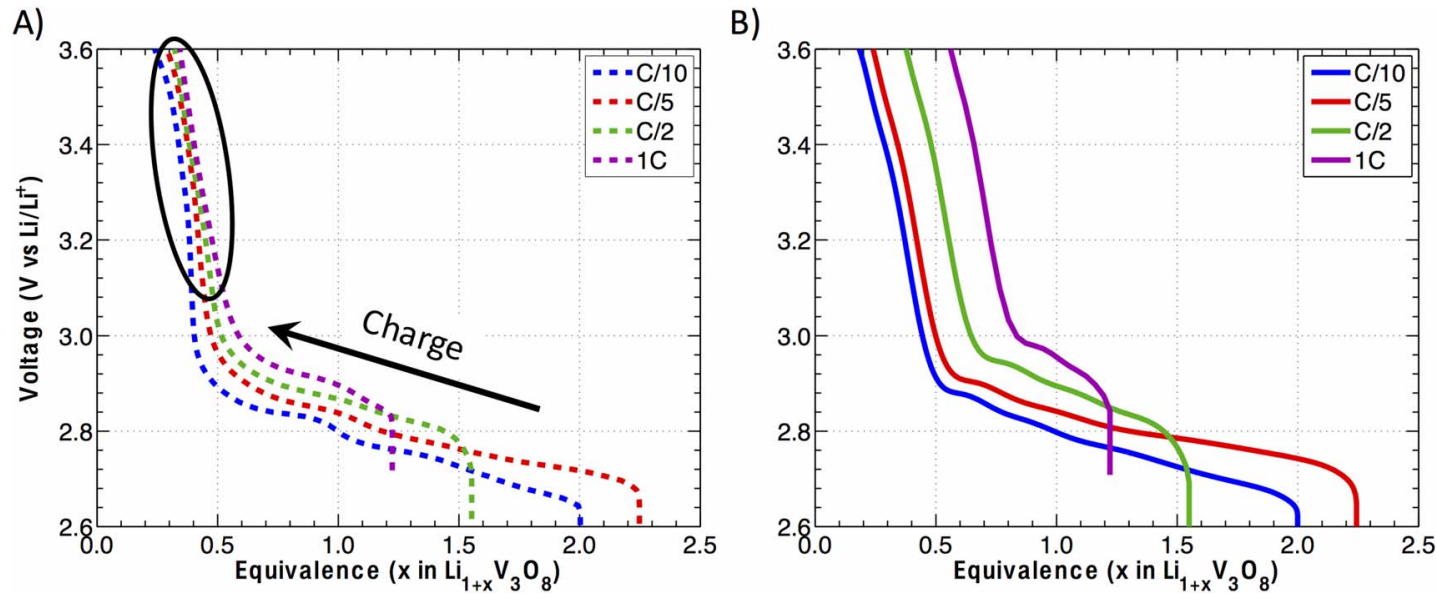

Figure 8. Experimental (A) potential during charge (delithiation) at different rates. The corresponding simulated (B) potential is shown for comparison. The simulations do not capture the relatively small changes in potential with charge rate observed at low equivalence.
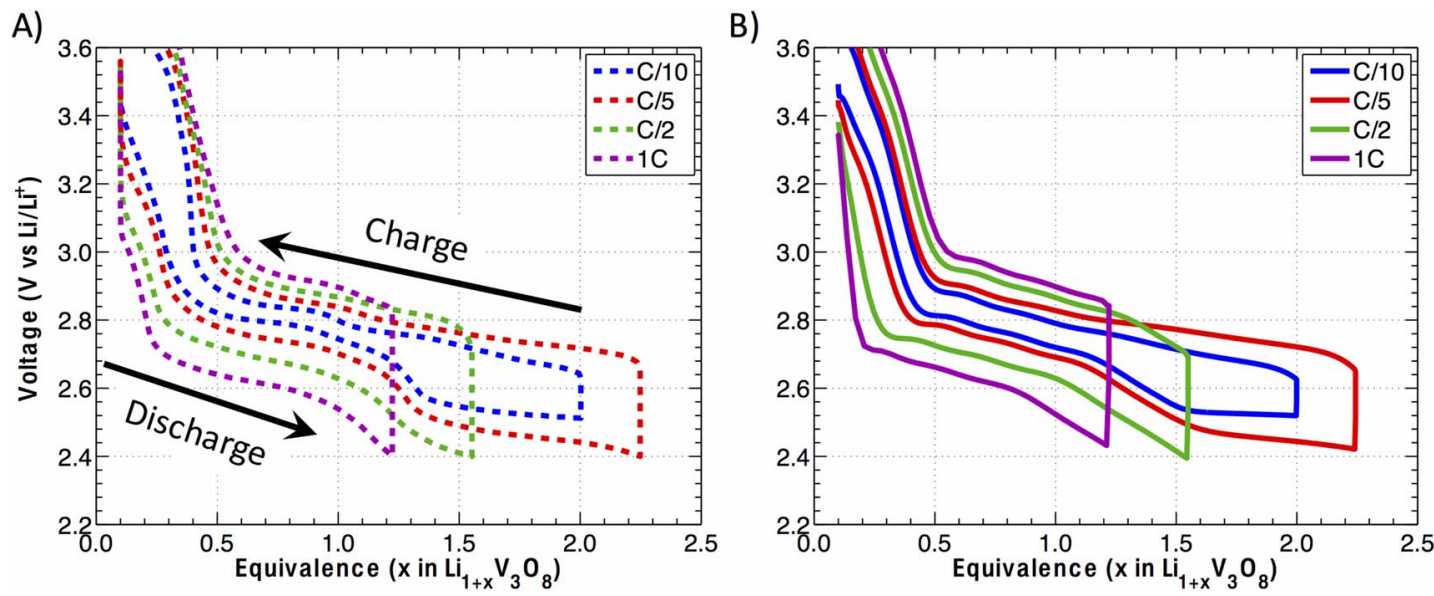

Figure 9. Experimental (A) potential during charge and discharge at different rates. The corresponding simulated (B) potential is shown for comparison. If the diffusion coefficient is increased during charge, then the simulation curves collapse onto each other as is observed experimentally, providing significant improvement from those observed in Figure 8.

lithiation. An alternative rationale is that the (100) face is an insulator to lithiation because surface adsorption energetics are not favorable. We speculate that both anisotropic transport and face-dependent kinetics may play a role.

Using the model we can examine the factors that contribute to voltage losses within the electrode. This is illustrated in Figure 10 current rate: $\mathrm{C} / 5$. The experimental data and model fit represent the potential between the lithium-metal anode and the vanadate cathode. The losses can be split into three components: losses due to chargetransfer, non-instantaneous phase change kinetics (supersaturation), and mass-transport. The open-circuit voltage is obtained by assuming instantaneous charge-transfer kinetics, phase change kinetics, and diffusive mass-transfer (i.e. it is assumed there are no voltage losses due to charge-transfer, phase-change, or mass-transport). The individual losses can be quantified by sequentially relaxing these ideal assumptions. Before phase change occurs the majority of the voltage drop occurs due to charge-transfer effects, but the losses due to masstransfer are also significant. At the end of the discharge, it is clear that all three effects present significant voltage drops to the system. Considering this information in the context of cell design, it suggests that decreasing the crystal size could significantly improve performance by decreasing the diffusion path-length thereby decreasing the mass-transfer resistance; additionally, the smaller crystal sizes would have a larger surface area to volume ratio, which would improve charge-transfer resistance. However, it is unclear how smaller crystals would impact phase-change kinetics and consideration needs to

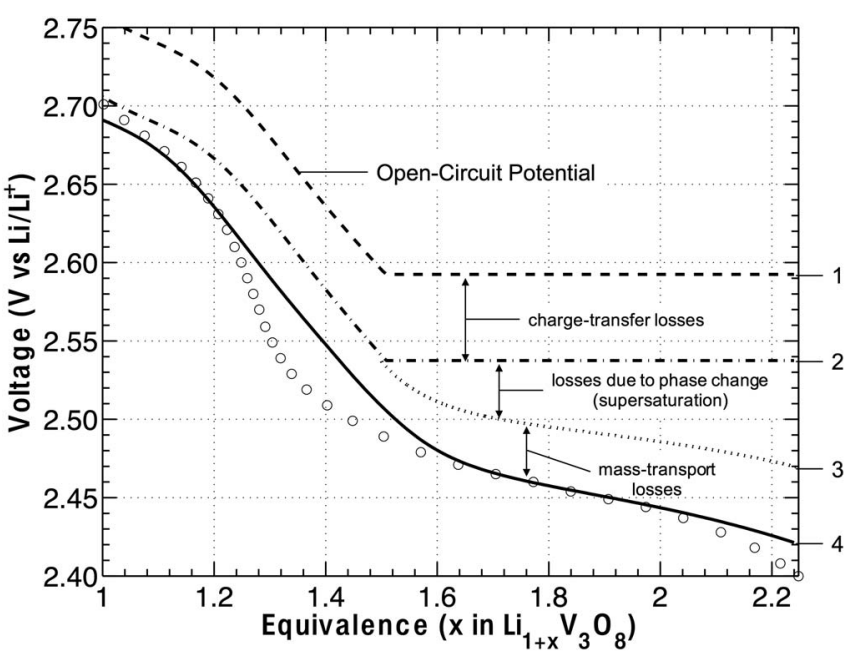

Figure 10. Estimation of the potential drops in the lithium trivanadate electrode at a current rate of $\mathrm{C} / 5$, focusing on the two-phase region. The graph shows qualitatively the contributions to the observed overpotential. 1) reversible potential, 2) charge-transfer resistance only, 3) charge-transfer and estimated phase-change resistances, assuming no concentration variations, 4) charge transfer, estimated phase-change, and estimated mass-transfer resistances. 
be given to how crystal size will affect cycling performance of this material.

It should be noted in Figure 10 that there is a slight discrepancy between the experimental data and the simulated voltage profiles in the range $\mathrm{x}=1.2$ to 1.6 in $\mathrm{Li}_{1+\mathrm{x}} \mathrm{V}_{3} \mathrm{O}_{8}$. While the exact reason for this mismatch is not known, the discrepancy occurs near the beginning of where phase change is predicted to occur, so it can be speculated that the discrepancy is affiliated with phase change. It is difficult to say whether the discrepancy is due to the assumed kinetic rate equation or additional physics such as strain effects which are not explicitly treated.

\section{Conclusions}

A combined experimental and theoretical study suggests that the phase transformation from $\alpha-\mathrm{Li}_{1+\mathrm{x}} \mathrm{V}_{3} \mathrm{O}_{8}$ to $\beta-\mathrm{Li}_{4} \mathrm{~V}_{3} \mathrm{O}_{8}$ is relatively facile. A model to analyze the electrochemical behavior and phase transformation also requires a description of mass transport of lithium in the crystal host material. Although SEM analysis reveals that the crystals aggregate, from the analysis of the time constant associated with voltage recovery it was concluded that the significant mass transfer resistances occur on the crystal scale and that these resistances are consistent with the assumption of 1-D diffusion along the [001] direction. Fitted phase-transformation kinetics suggest that $\psi_{T h} \sim 5$, and a shrinking-core model of phase distributions within a crystal is thus not appropriate. The excellent agreement between simulated and experimental lithiation results validates the selected diffusion coefficient of lithium in $\mathrm{LiV}_{3} \mathrm{O}_{8}$, as well as the selected value of the equilibrium concentration of lithium in the $\alpha$-phase. Comparisons also suggest that transport processes within the crystal may be more rapid during charge than discharge, from which we have hypothesized that the (001) crystal face may be active during charge but not during discharge. An analysis of the potential drop contributions indicates that losses due to charge-transfer, mass-transfer, as well as phase change are all significant.

\section{Acknowledgments}

The research was supported by the Center for Mesoscale Transport Properties, an Energy Frontier Research Center supported by the U.S. Department of Energy (DOE), Office of Science, Basic Energy Sciences (BES), under award \#DE-SC0012673. Microscopy conducted at the Center for Functional Nanomaterials at Brookhaven National Laboratory was supported by DOE-BES User Facility Division, under Contract No. DE-SC0012704. The DFT calculations were performed using computational resources at the Center for Functional Nanomaterials, a user facility at Brookhaven National Laboratory, under Contract No DE-SC0012704. K. W. K. greatly acknowledges the support of the National Science Foundation Graduate Research Fellowship under grant No. 1144155. Any opinions, findings, and conclusions or recommendations expressed in this material are those of the authors and do not necessarily reflect the views of the National Science Foundation.

\section{Appendix A}

This appendix details the mathematical model for the formulation of a new phase Equation [14] in the text, which is based on the formulations for nucleation and growth developed by Avrami. ${ }^{16-18,39}$ First it assumes that the radius, or characteristic length, of a nucleus grows at a constant rate, $v$, and can be described by Equation A1.

$$
r(t)=r^{*}+v t \approx v t
$$

where $r^{*}$ is the critical radius of a nucleus, and $t$ is time. Assuming spherical particles, the volume of a single nucleus is given by $\mathrm{A} 2$, and differentiating with respect to time gives A3.

$$
\begin{gathered}
V=\frac{4}{3} \pi r^{3}=\frac{4}{3} \pi v^{3} t^{3} \\
\frac{d V}{d t}=4 \pi v^{3} t^{2}=k_{g} V^{\frac{2}{3}}
\end{gathered}
$$

where $k_{g}$ is the growth rate constant.

The total volume of the $\beta$-phase is the product of the number of nuclei and the volume per nucleus, $V$. As such, the total growth rate of $\beta$-phase is the product of the growth rate of an individual nucleus and the number of nuclei:

$$
\frac{d V_{\beta}}{d t}=n \frac{d V}{d t}=n\left(4 \pi v^{3} t^{2}\right)
$$

As the nuclei grow they will begin to impinge upon each other. To ensure that there is no double counting, $n$ represents the number of isolated nuclei, given as $n_{0}\left[1-\theta_{\beta}\right]$, where $n_{0}$ is the total number of nuclei. As the volume fraction of $\beta$-phase increases, it becomes more likely that nuclei will impinge on each other, therefore decreasing the number of isolated nuclei. As super-saturation increases it is expected to favor nucleation, i.e. $\left(c_{\alpha}-c_{\alpha, \text { sat }}\right)$ is the driving force for nucleation. The growth rate, $v$, of an individual nucleus is assumed to be unaffected by super-saturation. Assuming progressive nucleation, $n$ can be represented using Equation A5

$$
n=\left(k_{n}\left(c_{\alpha}-c_{\alpha, s a t}\right) t\right)\left[1-\theta_{\beta}\right]
$$

where $k_{n}$ is the nucleation rate constant. Combining Equations A4 and A5 and dividing by the total volume, $V_{t o t}$, produces $\mathrm{A} 6$, which is a specific case of Equation 15, where $m=1$.

$$
\begin{aligned}
\frac{1}{V_{\text {tot }} \frac{d V_{\beta}}{d t}} & =\frac{1}{V_{\text {tot }}}\left(4 \pi v^{3} t^{3}\right) k_{n}\left(c_{\alpha}-c_{\alpha, \text { sat }}\right)\left[1-\theta_{\beta}\right] \\
& =\frac{1}{V_{\text {tot }}} k_{n}\left(c_{\alpha}-c_{\alpha, s a t}\right)(4 \pi V)\left[1-\theta_{\beta}\right] \\
\frac{d \theta_{\beta}}{d t} & =k_{\beta}\left(c_{\alpha}-c_{\alpha, s a t}\right)\left(\theta_{\beta}\right)\left[1-\theta_{\beta}\right]
\end{aligned}
$$

\section{Appendix B}

This appendix lists the open-circuit potential of excess lithium in lithium trivanadate $\left(\mathrm{LiV}_{3} \mathrm{O}_{8}\right)$. The expression for the open-circuit potential as a function of lithium concentration in the $\alpha$-phase is derived using the approach outlined by Karthikeyan et al. ${ }^{40}$ The open-circuit potential at a particular lithium concentration was estimated using

$$
\begin{aligned}
U= & U_{\text {ref }}+\frac{R_{G} T}{F} \ln \left[\left(\frac{c}{c_{0}}\right)\left(\frac{1-\bar{c}_{\max }}{\bar{c}_{\max }}\right)\right] \\
& +\sum_{k=0}^{N} A_{k}\left[\left(2 \bar{c}_{\max }-1\right)^{k+1}-\frac{2 \bar{c}_{\max } k\left(1-\bar{c}_{\max }\right)}{\left(2 \bar{c}_{\max }-1\right)^{1-k}}\right] \\
\bar{c}_{\max }= & \frac{c_{\alpha}}{c_{\alpha, \max }}
\end{aligned}
$$

\section{Table B1. Parameters for the Empirical Open Circuit Potential of the $\alpha$-Phase.}

\begin{tabular}{cc} 
Parameter & Value \\
\hline$N$ & 20 \\
$U_{\text {ref }}$ & 2.7671 \\
$A_{0}$ & -0.32895 \\
$A_{1}$ & 0.057048 \\
$A_{2}$ & -0.21475 \\
$A_{3}$ & 0.24177 \\
$A_{4}$ & 1.8186 \\
$A_{5}$ & -0.32144 \\
$A_{6}$ & -19.037 \\
$A_{7}$ & 11.997 \\
$A_{8}$ & 107.13 \\
$A_{9}$ & -111.70 \\
$A_{10}$ & -355.17 \\
$A_{11}$ & 489.45 \\
$A_{12}$ & 696.86 \\
$A_{13}$ & -1133.1 \\
$A_{14}$ & -813.10 \\
$A_{15}$ & 1438.6 \\
$A_{16}$ & 568.70 \\
$A_{17}$ & -953.47 \\
$A_{18}$ & -237.50 \\
$A_{19}$ & 260.21 \\
$A_{20}$ & 52.050 \\
$c_{0, m a x}\left(m_{01} \mathrm{mom}^{-3}\right)$ & 0.001 \\
& 0.0243
\end{tabular}




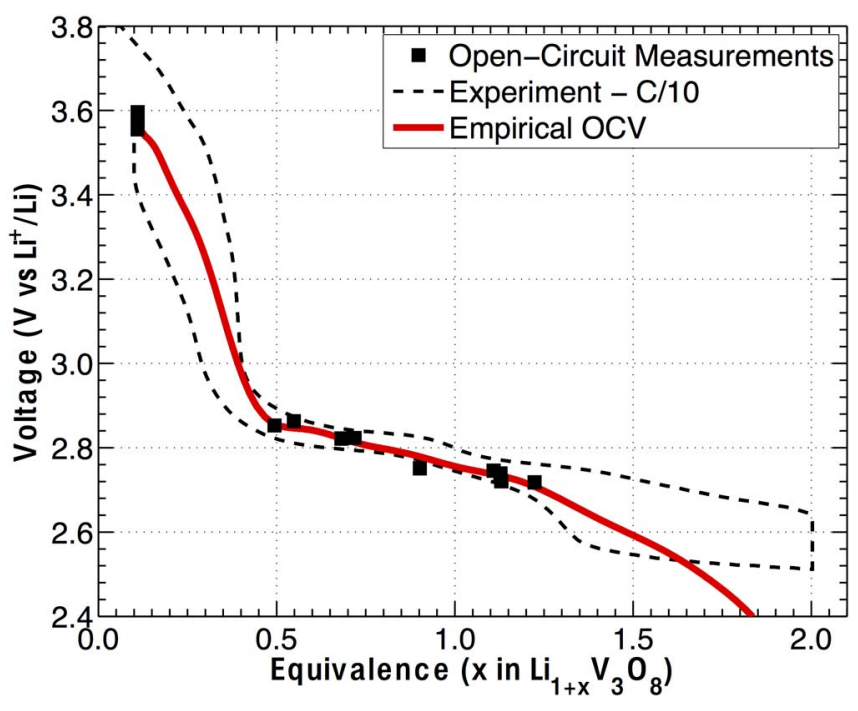

Figure B1. Open-circuit voltage measurements (squares) were taken and the empirical open-circuit voltage curve (solid red) was constructed to reside between the discharge and charge curve (dashed).

where $c$ is local concentration of lithium in the electrolyte. The parameters in Equation B1 are obtained by fitting the equation to experimental data and the values for the parameters are given in Table B1 and the fit is shown in Figure B1. The reason the empirical opencircuit voltage $(\mathrm{OCV})$ falls below the experimental electrochemical data $(\mathrm{x}=1.6$ in $\left.\mathrm{Li}_{1+\mathrm{x}} \mathrm{V}_{3} \mathrm{O}_{8}\right)$ is that the empirical OCV neglects the effects of phase change. The effects of phase change on the experimental data is to suppress the lithium concentration in the $\alpha$-phase, see Equation 6.

\section{List of Symbols}

$c_{0}$

$c_{\alpha, \max }$

$\bar{c}$

$D$

$D_{\text {eff }}$

F

$i$

$\bar{i}$

$i_{0}$

$i_{\text {app }}$

$k_{g}$

$k_{n}$

$k_{r x n}$

$k_{\beta}$

$L$

$m$

$n$ phase formation

$R_{G} \quad$ ideal gas constant $\left(8.314 \mathrm{~J} \mathrm{~mol}^{-1} \mathrm{~K}^{-1}\right)$

$r \quad$ particle radius $(\mathrm{cm})$

$r^{*} \quad$ critical particle radius $(\mathrm{cm})$

$T \quad$ Temperature $(\mathrm{K})$

$t \quad$ time (s)

$V \quad$ volume $\left(\mathrm{cm}^{3}\right)$

$V_{\text {tot }} \quad$ total volume $\left(\mathrm{cm}^{3}\right)$

$v \quad$ linear growth rate in Avrami formulation $\left(\mathrm{cm} \mathrm{s}^{-1}\right)$

$x \quad$ linear position in the crystal $(\mathrm{cm})$

$\bar{x} \quad$ dimensionless position

\section{Greek}

$\alpha_{a}, \alpha_{c} \quad$ anodic and cathodic charge-transfer coefficients

$\eta \quad$ overpotential $(\mathrm{V})$

$\theta \quad$ volume fraction of phase

$\rho \quad$ density of crystal material $\left(\mathrm{g} \mathrm{cm}^{-3}\right)$ $\tau \quad$ characteristic time $\left(L^{2} / D\right)$

$\psi_{T h} \quad$ ratio of rate of phase transformation to diffusive mass transfer rate

\section{Subscripts}

$\alpha$

$\beta$

$C T$

$g b$

$M T$

denotes the $\alpha$-phase (alpha-phase)

denotes the $\beta$-phase (beta-phase)

charge-transfer

grain-boundary

mass-transfer

\section{References}

1. H. Wu and Y. Cui, Nano Today, 7, 414 (2012).

2. M. S. Whittingham, Chemical Reviews, 104, 4271 (2004).

3. S. Panero, M. Pasquali, and G. Pistoia, Journal Electrochemical Society: Accelerated Brief Communication, 130, 1225 (1983).

4. M. E. Spahr, P. Novak, W. Scheifele, O. Haas, and R. Nesper, Journal of The Electrochemical Society, 145, 421 (1998).

5. N. A. Chernova, M. Roppolo, A. C. Dillon, and M. S. Whittingham, Journal of Materials Chemistry, 19, 2526 (2009).

6. A. Pan, J. Liu, J.-G. Zhang, G. Cao, W. Xu, Z. Nie, X. Jie, D. Choi, B. W. Arey, C. Wang, and S. Liang, Journal of Materials Chemistry, 21, 1153 (2011).

7. M. Winter, J. O. Besenhard, M. E. Spahr, and P. Novák, Advanced Materials, 10, 725 (1998).

8. S. Sarkar, A. Bhowmik, M. Dixit Bharadwaj, and S. Mitra, Journal of The Electrochemical Society, 161, A14 (2013).

9. G. Pistoia, M. Pasquali, M. Tocci, R. V. Moshtev, and V. Maner, Journal of The Electrochemical Society, 132, 281 (1985).

10. L. A. Picciotto, K. T. Adendorff, D. C. Liles, and M. M. Thackeray, Solid State Ionics, 62, 297 (1993).

11. S. Jouanneau, A. Verbaere, and D. Guyomard, Journal of Solid State Chemistry, 178, 22 (2005).

12. V. Srinivasan and J. Newman, Journal of The Electrochemical Society, 151, A1517 (2004).

13. A. S. Andersson and J. O. Thomas, Journal of Power Sources, 97, 498 (2001).

14. C. Delmas, M. Maccario, L. Croguennec, F. Le Cras, and F. Weill, Nature Materials, 7, 665 (2008).

15. L. Laffont, C. Delacourt, P. Gibot, M. Yue Wu, P. Kooyman, C. Masquelier, and J. M. Tarascon, Chemistry of Materials, 18, 5520 (2006).

16. M. Avrami, The Journal of Chemical Physics, 7, 1103 (1939).

17. M. Avrami, The Journal of Chemical Physics, 8, 212 (1940).

18. M. Avrami, The Journal of Chemical Physics, 9, 177 (1941).

19. J. L. Allen, T. R. Jow, and J. Wolfenstine, Chemistry of Materials, 19, 2108 (2007).

20. N. W. Brady, K. W. Knehr, C. A. Cama, C. N. Lininger, Z. Lin, A. C. Marschilok, K. J. Takeuchi, E. S. Takeuchi, and A. C. West, Journal of Power Sources, 321, 106 (2016).

21. A. C. Marschilok, S. H. Lee, C. C. Milleville, P. Chen, E. S. Takeuchi, and K. J. Takeuchi, Journal of Composite Materials, 47, 33 (2013).

22. J. P. Perdew and Y. Wang, Physical Review B, 45, 13244 (1992).

23. G. Kresse and J. Furthmuller, Physical Review B, 54, 11169 (1996).

24. A. Jain, G. Hautier, C. J. Moore, S. Ping Ong, C. C. Fischer, T. Mueller, K. A. Persson, and G. Ceder, Computational Materials Science, 50, 2295 (2011).

25. H. J. Monkhorst and J. D. Pack, Physical Review B, 13, 5188 (1976).

26. M. M. Thackeray, W. I. F. David, and J. B. Goodenough, Materials Research Bulletin, 17, 785 (1982).

27. T. Jiang and M. L. Falk, Physical Review B, 85 (2012).

28. K. W. Knehr, N. W. Brady, C. A. Cama, D. C. Bock, Z. Lin, C. N. Lininger, A. C. Marschilok, K. J. Takeuchi, E. S. Takeuchi, and A. C. West, Journal of The Electrochemical Society, 162, A2817 (2015).

29. K. W. Knehr, N. W. Brady, C. N. Lininger, C. A. Cama, D. C. Bock, Z. Lin, A. C. Marschilok, K. J. Takeuchi, E. S. Takeuchi, and A. C. West, ECS Transactions, 69, 7 (2015).

30. A. D. Wadsley, Acta Crystallographica, 10, 261 (1957).

31. N. Kumagai and A. Yu, Journal of The Electrochemical Society, 144, 830 (1997).

32. J. Newman and Karen E. Thomas-Alyea, Electrochemical Systems, John Wiley \& Sons Inc., Hoboken, New Jersey (2004).

33. K. W. Knehr, C. A. Cama, N. W. Brady, A. C. Marschilok, K. J. Takeuchi, E. S. Takeuchi, and A. C. West, Electrochimica Acta, (Submitted) (2016).

34. G. Pistoia, M. L. Di Vona, and P. Tagliatesta, Solid State Ionics, 24, 103 (1987).

35. I. D. Raistrick, Revue de Chimie Minerale, 21 (1984).

36. A. Hammou and A. Hammouche, Electrochimica Acta, 33, 1719 (1988).

37. J. Kawakita, Y. Katayama, T. Miura, and T. Kishi, Solid State Ionics, 110, 199 (1998).

38. G. Chen, X. Song, and T. J. Richardson, Electrochemical and Solid-State Letters, 9 , A295 (2006).

39. A. K. Jena and M. C. Chaturvedi, Phase transformation in materials, p. 243, Prentice Hall (1992).

40. D. K. Karthikeyan, G. Sikha, and R. E. White, Journal of Power Sources, 185, 1398 (2008). 